\title{
Symptomkontrolle steht bei älteren Diabetikern im Fokus
}

\author{
Die Diabetestherapie sollte altersabhängig erfolgen. \\ Je hilfsbedürftiger Senioren mit Diabetes sind, desto \\ mehr stehen die reine Prävention diabetesbedingter \\ Symptome und die strikte Vermeidung von \\ Hypoglykämien im Fokus. Besonderes Augenmerk \\ sollte bei Älteren auch auf die Komorbidiät und das \\ Interaktionsrisiko bei Polypharmazie gelegt werden.
}

Ältere Diabetiker sind eine besonders uneinheitliche Patientengruppe, betonte Dr. Alexander Friedl, Ärztlicher Leiter des geriatrischen Zentrums Stuttgart. Es gebe sehr fitte, nicht geriatrische Patienten, so genannte Go-Go's, bei denen ähnlich wie bei jüngeren eine leitliniengerechte Diabetestherapie mit primär- und sekundärpräventivem Ansatz erfolgen sollte. Haben die Patienten leichte Handicaps und sind hilfsbedürftig (Slow-Go's), sind die wichtigsten Behandlungsziele Erhalt bzw. Steigerung der Selbstständigkeit und Lebensqualität. Haben sie schwere Handicaps und sind im Alltag weitgehend hilflos (No-Go`s), sollten unter Einbeziehung der individuellen Patientenwünschen vor allem die Symptome kontrolliert werden. Der $\mathrm{HbA}_{1 \mathrm{c}}$-Wert sollte jedoch auch bei Älteren einen Wert von 8,0\% nicht überschreiten, sagte Friedl, die Untergrenze liege bei 6,5\%. Sinke bei einem älteren $\mathrm{Pa}$ tienten das $\mathrm{HbA}_{1 \mathrm{c}}$ zu tief, sollte eine mögliche Anämie abgeklärt werden, betonte der Geriater und Diabetologe. Denn bei anämischen Patienten würden falsch-niedrige $\mathrm{HbA}_{1 \mathrm{c}}$-Werte gemessen.

Bei allen älteren Diabetikern sollten zur Abklärung häufiger geriatrischer Syndrome u.a. Mobilität, Ernährung, Kognition und Psyche strukturiert beurteilt werden, so Friedl weiter. Gang- und Sehstörungen, Schwindel und Exsikkosen tragen häufig zum hohen Sturz- und Frakturrisiko bei Älteren bei. Das strikte Vermeiden von Hypoglykämien ist v.a. prognostisch relevant, aber in der Praxis gar nicht einfach. „Unterzuckerungen sind bei Älteren schwieriger zu erkennen, weil sie weniger Symptome haben“, betonte Friedl. Aber: Hyperglykämien korrelieren klar mit einer erhöhten Mortalität, wie in Studien z.B mit Sulfonylharnstoffen (SH) belegt worden ist. Zudem erhöht sich das Demenzrisiko deutlich, sagte die Pharmazeutin Mag.pharm. Martina Anditsch vom Allgemeinen Krankenhaus Wien.

\section{Wechselwirkungen beachten}

Problematisch sind oft die Medikamentencocktails bei multimorbiden Diabetikern, auch in Bezug auf das HypoglykämieRisiko. Anditsch warnte etwa vor der Kombination von Chinolonen mit SH, bei der das Hypoglykämie-Risiko deutlich erhöht sei. Denn Chinolone führen über einen verstärkten Kalziumeinstrom zu einer Insulinfreisetzung, die bei Diabetikern nicht kompensatorisch gegenreguliert werden könne. Auch viele weitere, bei Älteren häufig eingesetzten Arzneien wie das Gichtmittel Allopurinol fördern Hypoglykämien. Umgekehrt schwächen andere Medikamente die Wirkung von Antidiabetika ab - An-

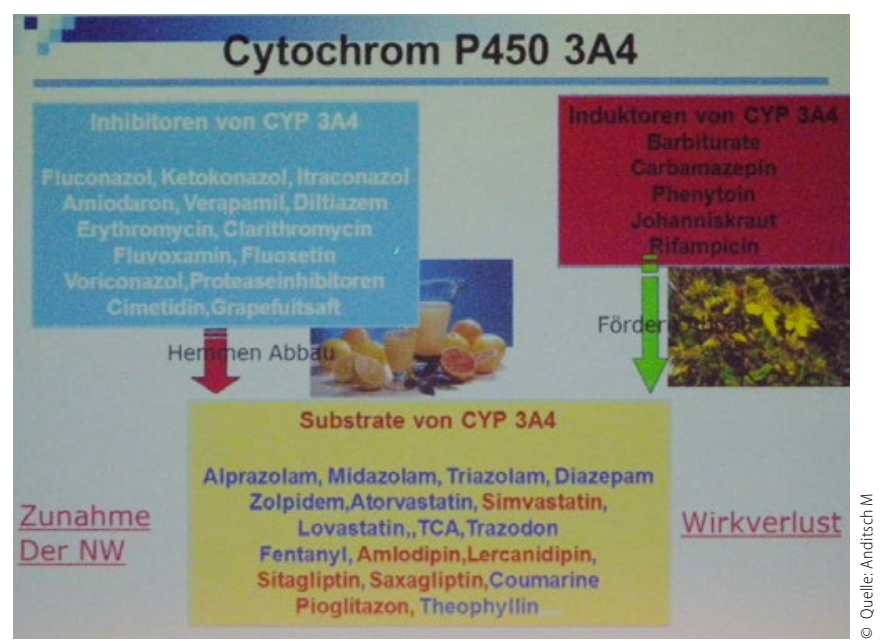

1 Vorsicht, viele Wirkstoffe interagieren mit CYP3A4.

ditsch nannte Schilddrüsenmedikamente, Präparate weiblicher Hormon, Diuretika, Kortikosteroide, Antipsychotika, Antiepileptika-, sodass die Gefahr von Hyperglykämien verstärkt wird.

Eine Langzeittherapie mit Metformin ist nach Angaben der Pharmazeutin öfter mit einem Vitamin $B_{12}$-Mangel verbunden, einem Risikofaktor für Polyneuropathien. Das Antidiabetikum hemme vermutlich die Resorption eines Enzyms. Geachtet werden sollte bei multimorbiden Patienten zudem auf Elektrolytstörungen wie Hyperkaliämien und Hyponatriämien. Bei Diabetikern sei die Aufnahme von Kalium in die Zelle verringert und viele Medikamente wie RAS-Hemmer, Betablocker und Heparine erhöhen Kaliumspiegel, andere wie NSAR verringern die renale Kaliumausscheidung. Eine Hyperkaliämie sei die Folge, die zu Herzrhythmusstörungen, Synkopen und zum akuten Nierenversagen bis zur Dialyse führen könne, so Anditsch.

Eine Hyponatriämie droht u. a. bei Diuretikatherapie, vor allem mit Thiaziden und Schleifendiuretika, auch bei medikamentöser Beeinträchtigung der ADH-Sekretion, z. B. durch Antipsychotika, Antidepressiva und Benzodiazepine. Auch SGLT2-Hemmer erhöhen die Natriumausscheidung und sollten daher bei Patienten unter Diuretika sehr vorsichtig eingesetzt werden, sagte Anditsch.

Last but not least: Bei einer Polypharmazie gibt es oft Interaktionen über Cytochrom-P450-3A4, über das zum Beispiel Gliptine, Kalzium-Antagonisten und Statine verstoffwechselt werden $(\triangleright$ Abb. 1). Generell sollte laut Anditsch von Grapefruitsaft, einem CYP3A4-Hemmer, abgeraten werden. Zu Hemmstoffen des Enzyms zählen zudem Pilzmittel und Makrolid-Antibiotika, zu Induktoren, die zum Wirkverlust der Substate führen, u.a. Barbiturate, Johanniskraut und Rifampicin.

Roland Fath

Quelle: DDG-Herbsttagung 2015. 6.-7. November 2015, Düsseldorf 